\title{
Glanzmann thrombasthenia and its perioperative management in head trauma: A rare and challenging situation
}

\author{
Neeraj Kumar, Abdul A. Khan, Nilesh Kumar', Ashish Bindra, Charu Mahajan, Keshav Goyal
}

\begin{abstract}
Glanzmann thrombasthenia is a rare inherited coagulation disorder, manifesting itself in early childhood as bleeding episodes.The patients with this disease may present with uncontrollable bleeding following trauma. We report a patient of Glanzmann syndrome with head injury, who presented to us following fall from stairs. Platelet transfusion is the mainstay of treatment in emergency situations.As patients generally have a history of platelet transfusion they may be refractory to the same. Leuko reduced or human leukocyte antigen matched platelets should be used. Recombinant factorVIla can be used to control bleeding in refractory cases and in patients with antibodies against platelets.Anti-fibrinolytics can also be used as adjuvants. There is need of advanced perioperative coagulation screening spot tests in emergency trauma settings as routine coagulation tests may not be able to pick up the abnormality. Thromboelastography/thromboelastometry can be a great tool for perioperative management of such coagulation disorders.
\end{abstract}

Key words: Anaesthesia, glanzmann thrombasthenia, head injury, perioperative, platelets, thromboelastography

\section{INTRODUCTION}

Bleeding following trauma is a big menace more so in patients who have defective coagulation. Glanzmann thrombasthenia (GT) is a rare autosomal recessive bleeding syndrome affecting megakaryocyte lineage and is characterized by a lack of platelet aggregation. It is caused by deficiency or abnormality of platelet membrane glycoprotein (GP) IIb and/or IIIa. The platelet count is normal or subnormal, but bleeding time (BT) is prolonged due to defective or absent platelet aggregation. It is a rare disorder with an incidence of about 1/1.000.000, but can be seen commonly in populations where consanguineous

\begin{tabular}{|l|l|}
\hline \multicolumn{2}{|c|}{ Access this article online } \\
\hline Quick Response Code: & Website: \\
\hline & www.jnaccjournal.org \\
\cline { 2 - 2 } & \\
\hline & DOI: \\
\hline
\end{tabular}

marriages are frequent. ${ }^{[1-3]}$ The clinical manifestation is generally mucocutaneous bleeding varying in severity. It may manifest as spontaneous epistaxis, gum bleeding, easy bruising, purpura, menorrhagia, gastrointestinal bleeding, haematuria, muscle haematoma, and traumatic or postoperative bleeding with a normal platelet count. Central nervous system and visceral haemorrhages may sometimes be encountered but are not commonly reported. ${ }^{[4]}$ The platelets of these patients cannot bind fibrinogen and aggregation does not occur. Though bleeding tendency varies in frequency and severity, but traumatic and postoperative bleedings pose a special challenge. We report a case of head trauma with Glanzmann syndrome, where haemorrhage control in acute and emergency settings became very difficult.

\section{CASE REPORT}

A 16-year-old female, born out of non-consanguineous marriage presented with a history of fall from stairs in morning hours, which was not associated with the loss of consciousness, vomiting or seizures. Patient was

Departments of Neuroanaesthesiology and Critical Care and ${ }^{1}$ Anaesthesiology, All India Institute of Medical Sciences and Associated Jai Prakash Narain Apex Trauma Centre, New Delhi, India 
conscious and oriented after the incident and managed to get up on her own. However, $2 \mathrm{~h}$ later patient started complaining of headache, which gradually increased in severity for which she was admitted to the emergency department of our hospital $3 \mathrm{~h}$ after fall. Admission Glasgow Coma score was E4V5M6 but soon after admission patient became drowsy with GCS deteriorating to E1V1M5. The patient was intubated, stabilized and a noncontrast computed tomogram brain was obtained, which revealed right fronto-temporo-parietal acute subdural haematoma, high parietal extra-dural haematoma along with right subarachnoid haemorrhage. There was a midline shift of $4 \mathrm{~mm}$ [Figure 1].

History was significant for development of petechial spots all over the body off and on, easy bruisability, mucosal gum bleeding and heavy menstrual bleeding. Patient was diagnosed as GT at the age of 8 years and was treated intermittently with platelet transfusions. Previous hematological studies revealed a platelet count of $195 \times 10^{3} / \mathrm{mm}^{3}$, clotting time $6 \mathrm{~min}$, BT $16 \mathrm{~min}$, prothrombin time (PT) 13/12 and activated partial thromboplastin time (aPTT 31/30). Platelet function test showing PF3 (platelet factor) availability with adenine diphosphate (ADP) $28 \mathrm{~min} / 24 \mathrm{~min}$, total PF3 9/7. Platelet aggregation was normal with ristocetin, but absent with epinephrine, ADP, and collagen. Flow cytometry was suggestive of platelet function disorder and negative CD-41 (gpIIb) and CD-61 (gpIIIa). These findings confirmed the diagnosis of GT. There was also history of iron deficiency anaemia with serum ferritin $<10 \mathrm{mg} / \mathrm{ml}$, serum iron $48 \%$ and total iron binding capacity $348 \%$ and transferrin saturation $13.7 \%$. There was no history of similar bleeding tendency in the family.

Patient was taken to intensive care unit, where Codman intraparenchymal, intracranial pressure (ICP) monitoring was started. However during insertion of ICP catheter

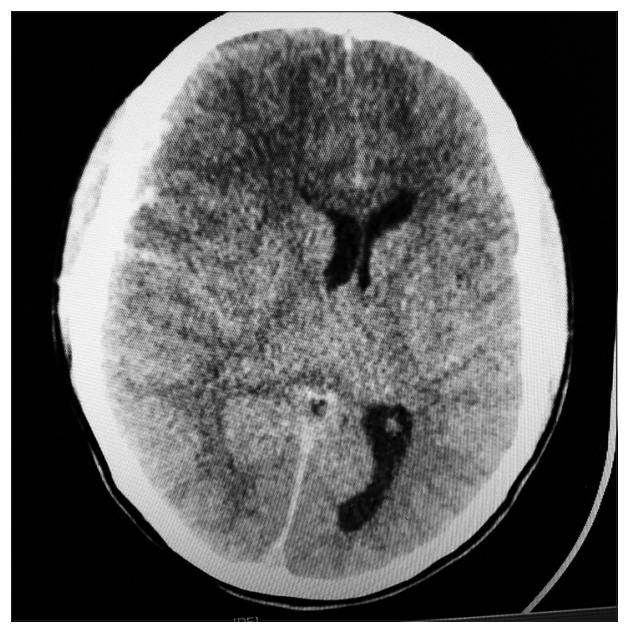

Figure 1: Preoperative noncontrast computed tomogram head excessive bleeding was present which stopped after compression. The opening ICP was $77 \mathrm{mmHg}$. In view of persistently raised ICP and deteriorating neurological status an emergency decompressive craniectomy was planned. Laboratory tests revealed normal PT (16.3/14 s), aPTT (29.2 s), international normalized ratio (INR) (1.31) and platelet count $\left(270 \times 10^{3} / \mathrm{mm}^{3}\right)$. BT (15 min) was prolonged. Other preoperative investigations were unremarkable, $\mathrm{Hb} 14.6 \mathrm{~g} / \mathrm{dL}, \mathrm{Na}$ $138 \mathrm{mEq} / \mathrm{L}$, K $4.0 \mathrm{mEq} / \mathrm{L}$, TLC $24560 / \mathrm{mm}^{3}$, INR 0.99, urea/creatinine 32/0.7. Arterial blood gas analysis showed pH 7.35, $\mathrm{PO}_{2}$ 92, $\mathrm{PCO}_{2} 35 \mathrm{mmHg}, \mathrm{HCO}_{3}^{-}$19.3. However, thromboeleastography (TEG) showed R: $7.5 \mathrm{~mm}, \mathrm{~K}: 9.8 \mathrm{~min}$, alpha angle: $33.23^{\circ}$, maximum amplitude (MA):100.4 mm, time to maximal amplitude: 87. $54 \mathrm{~min}$. The results of TEG did not correlate well with the clinical condition and increased BT. Six units of platelets were transfused preoperatively. Single donor platelets (SDPs), packed red blood cells and fresh frozen plasma was arranged, and the patient was taken to operation room for decompressive craniectomy ( $2 \mathrm{~h}$ postadmission). Inside operation room, standard monitoring including an electrocardiogram (ECG), pulse oximetry $\left(\mathrm{SpO}_{2}\right), \mathrm{ETCO}_{2}$ and noninvasive blood pressure (NIBP) was attached. Preoperative vitals included a heart rate of 109 beats/min, NIBP $138 / 88 \mathrm{mmHg}$, and $\mathrm{SpO}_{2} 98 \%$. Induction of anaesthesia was done with fentanyl $(2 \mathrm{mcg} / \mathrm{kg}$ ) and propofol $(80 \mathrm{mg})$. Mechanical ventilation was started, and anaesthesia was maintained with sevoflurane (1.0 to - 1.2 MAC) in $\mathrm{O}_{2}$ : Air $\left(\mathrm{FiO}_{2}\right.$ 0.5). Rocuronium was given intermittently for muscle relaxation. Right Internal jugular vein was cannulated with a double lumen 7 Fr catheter under ultrasound guidance in first attempt. Right radial artery was cannulated for invasive blood pressure (IBP) monitoring. Normal saline was used for intraoperative volume replacement. Intraoperative blood salvage was done using cell saver (Medtronic autolog, USA) for autologus blood transfusion. Total blood loss was around $2 \mathrm{~L}$ and total duration of surgery was $4.5 \mathrm{~h}$. Along with fluid resuscitation with crystalloids, $2 \mathrm{U}$ of red blood cells, $600 \mathrm{ml}$ of autologus blood from cell saver, $4 \mathrm{U}$ fresh frozen plasma was transfused. One gram, intravenous tranexemic acid was given intravenously. The mean arterial pressure was kept between 60 and $70 \mathrm{mmHg}$, on noradrenaline infusion.

In view of poor neurological status, patient was shifted to intensive care unit. Neuromuscular blockade was not reversed in postoperative period. The patient was on nor adrenaline, $8 \mathrm{mcg} / \mathrm{min}$ with following vitals HR: 150 bpm, BP: 100/50 mmHg, SpO2 99\%. She was electively ventilated on volume control mode titrated to $\mathrm{PaCO} 2$ level $30-35 \mathrm{mmHg}$. As a standard protocol, for sedation and analgesia titrated midazolam and fentanyl infusion was started. Postoperative monitoring 
included ECG, NIBP, IBP, central venous pressure, IBP, ICP and ETCO2. her postoperative hematological and biochemical parameters were $\mathrm{Hb}: 12.7 \mathrm{~g} / \mathrm{dl}, \mathrm{Na}: 157$ $\mathrm{mEq} / \mathrm{L}, \mathrm{K}+: 4.8 \mathrm{mEq} / \mathrm{L}$ TLC: $15300 / \mathrm{mm} 3$, Urea: 28 $\mathrm{mg} / \mathrm{dl}$, serum creatine: $0.6 \mathrm{mg} / \mathrm{kg}$, INR: 1.31 , Platelets: $102 \times 103 / \mathrm{mm} 3$, PT 13.7/16.9, BT 10 min. patient did not show any neurological improvement and despite all treatment ICP was persistently high $(>50 \mathrm{mmHg})$. The postoperative $\mathrm{CT}$ scan revealed new haematoma and diffuse cerebral oedema [Figure 2]. A postoperative TEG indicated the requirement of platelets for which another $4 \mathrm{U}$ of platelets were transfused. However, patient did not show any improvement and was declared brain dead on 1st postoperative day. However, patient didn't show any improvement and was declared brain dead on 1st postoperative day followed by death on 2 nd postoperative day.

\section{DISCUSSION}

Glanzmann's thrombasthenia is a rare inherited disorder of platelet function characterized by bleeding episodes. The laboratory studies show prolonged BT with absent or decreased platelet retraction in presence of a normal platelet count. Other coagulation studies are normal. Diagnosis is suspected in cases with repeated episodes of mucocutaneous bleeding and is confirmed by platelet aggregation studies. Platelet aggregation is absent in response to all physiological stimuli despite of normal count. Essential diagnostic features normal platelet count and morphology, prolonged BT, absence of platelet aggregation in response to ADP, collagen, epinephrine, thrombin and to all other aggregating agents. It is because of defective fibrinogen binding to platelets. Flow cytometry, studies of GPIIb-IIIa receptors on platelet membrane surface using monoclonal antibodies can also be carried out. ${ }^{[2,3]}$ Screening for platelet antibodies in patients with previous history

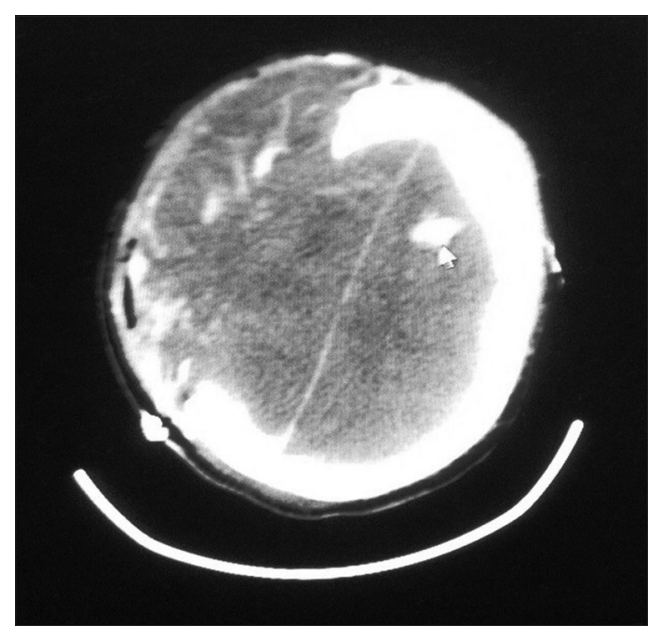

Figure 2: Postoperative noncontrast computed tomogram head (arrow showing the tip of intracranial pressure probe) of transfusion should be done. However, these test are carried out in sophisticated laboratory, generally under elective settings. In emergency settings, they may not be available and feasible. Furthermore in acute and ongoing blood loss as in trauma it may not be as useful because of its inability to show trends of coagulation.

There are three variants of GT, depending upon the availability of GPIIb/IIIa. However severity of bleeding is not correlated with the subtype. Haemorrhage is naturally the main clinical concern and supportive care is critical. The minor bleeding episodes can be controlled by local pressure, nasal packing with gelatin sponge for epistaxis, local haemostatics (e.g. fibrin glue and topical thrombin), as well as oral anti-fibrinolytics. However, in nonresponders and those with severe bleeding, platelet transfusions are recommended as standard of treatment. In patients receiving repeated transfusions antibodies against GPIIb/IIIa and/or human leukocyte antigens (HLAs) are formed which result in a variable degree of refractoriness to further platelet transfusions. As these patients require multiple transfusions during their lifetime, they should receive leukocyte-depleted blood products, in order to avoid platelet alloimmunization. Platelets matched via HLA can also prevent alloimmunization. Medications that affect platelet function, such as aspirin and other nonsteroidal anti-inflammatory drugs should be avoided. Although the superiority of SDP over pooled platelet transfusion is not well-established, preparation of SDPs appears prudent and may assist in management of these patients. Usually in emergency trauma settings facilities for HLA matching or leuco-reduction are unavailable. In our trauma center too we did not have this facility. Under such circumstances we gave filtered PRP in the perioperative period. Normal blood transfusion set with a filter of 170 microns can filter leucocytes and should be used for platelet transfusion.

Recombinant activated coagulation factor VIIa (NovoSeven ${ }^{\circledR}$; Novo Nordisk A/S, Malov, Denmark) has been used with variable success to control bleeding episodes in patients with GT. It was initially developed to treat bleeding episodes in patients with congenital haemophilia, but has been approved for the treatment of bleeding in patients with haemophilia A and B, acquired haemophilia, factor VII deficiency. ${ }^{[4,5]}$ It represents an alternative in cases with antibodies and/or a history of refractoriness to platelet transfusion. It is often used in association with anti-fibrinolytic agents. Thromboembolic events are a rare but potential hazard. The efficacy of factor VII in this situation is mostly attributable to enhanced platelet-surface FXa generation. It bypasses factor VIIIa and IXa and provides production of factor X, which activates coagulation system, and thereby provides haemostasis. ${ }^{[6]}$ The recommended rFVIIa bolus dose is of 
$90 \mathrm{mg} / \mathrm{kg}$ at $2 \mathrm{~h}$ intervals until hemostasis is achieved. However, its use should be judicious because of associated risk of thrombosis. In our case, it was not available.

Desmopressin has been used with unequivocal success in patients with defective platelet adhesion, however, its role in platelet aggregation abnormalities is not established. Anti-fibrinolytics can be used as an adjunct in preventing/controlling bleeding. Tranexamic acid or aminocaproic acid have been used to control bleeding episodes. ${ }^{[7]}$

For elective surgical procedure, it is important to plan major procedures with good communication between surgeons, anaesthesiologist and transfusion laboratory. Blood sampling for HLA antibodies and anti-platelet antibodies is recommended. Preoperative anti-fibrinolytics can be started. Platelet transfusion should be given immediately prior to the procedure, and further transfusion must be based on clinical need. rVIIa can also be given for $48 \mathrm{~h}$ postoperatively as an adjunct. ${ }^{[4,7]}$

In our case, the patient was a known case of GT, with a with a history of multiple platelet transfusions in past. She presented with deteriorating GCS but was haemodynamically stable initially without active bleeding from any other site. Coagulation studies were normal except for prolonged BT. Perioperative TEG can prove to be an invaluable tool for bedside monitoring of coaglutation status in such cases ${ }^{[8]}$ TEG provides global information on the dynamics of clot development, stabilisation and dissolution that reflect in vivo haemostasis, thus can enable the anaesthesiologist to keep a track of rapidly altering hemostatic profile. Decreased MA indicates a need for platelet transfusion. The use of blood compounds and pharmacological agents can be judiciously planned with it. Though the use of thromboelastography/thromboeleastometry has been recommended in patients with coagulation disorders, ${ }^{[9]}$ in our patient we could not find platelet deficiency on first TEG, possibly because it was done early after admission or due to processing error. Therapeutic effect of thrombocyte transfusion and anti-fibrinolytics has also been assessed by TEG in such conditions. However in a study by Lak et al. rotational thromboelestometry was not found to be beneficial for monitoring rFVIIa therapy in GT patients. ${ }^{[10]}$
Unfortunately due to severity of the injury and suboptimally controlled intracranial bleed we lost our patient. The purpose of reporting this case is to discuss the management of patients with rare inherited bleeding disorder in emergency trauma settings. To best of our knowledge, there is scant literature regarding the management of GT with severe traumatic brain injury. We want to highlight the need for advanced perioperative coagulation screening spot tests in emergency trauma settings as routine coagulation tests may not be able to pick up abnormalities.

\section{REFERENCES}

1. Glanzmann E. Hereditaire hamorrhagische thrombasthenic. Ein beitrag zur pathologie der blutplattchen. Jahrb Kinderheilkd 1918;88:1-42.

2. Nair S, Ghosh K, Kulkarni B, Shetty S, Mohanty D. Glanzmann’s thrombasthenia: Updated. Platelets 2002;13:387-93.

3. Kannan M, Saxena R. Glanzmann's thrombasthenia: An overview. Clin Appl Thromb Hemost 2009;15:152-65.

4. Franchini M, Lippi G. NovoSeven (recombinant factor VIla) for the treatment of bleeding episodes and perioperative management in patients with Glanzmann's thrombasthenia. Expert Rev Hematol 2014;7:733-40.

5. Kessler CM. New products for managing inhibitors to coagulation factors: A focus on recombinant factor VIla concentrate. Curr Opin Hematol 2000;7:408-13.

6. Ovanesov MV, Panteleev MA, Sinauridze EI, Kireev DA, Plyushch OP, Kopylov KG, et al. Mechanisms of action of recombinant activated factor VII in the context of tissue factor concentration and distribution. Blood Coagul Fibrinolysis 2008;19:743-55.

7. Alamelu J, Liesner R. Modern management of severe platelet function disorders. Br J Haematol 2010;149:813-23.

8. Topal A, Kılıçaslan A, Erol A, Çankaya B, Otelcioğlu S. Anaesthetic management with thromboelastography in a patient with Glanzmann thrombasthenia. Turk J Anaesth Reanim 2014;42:227-9.

9. Chitlur M, Rivard GE, Lillicrap D, Mann K, Shima M, Young G, et al. Recommendations for performing thromboelastography/ thromboelastometry in hemophilia: Communication from the SSC of the ISTH. J Thromb Haemost 2014;12:103-6.

10. Lak M, Scharling B, Blemings A, Sharifian R, Maleki Z, Daraee A, et al. Evaluation of rFVIIa (NovoSeven) in Glanzmann patients with thromboelastogram. Haemophilia 2008;14:103-10.

How to cite this article: Kumar N, Khan AA, Kumar N, Bindra A, Mahajan C, Goyal K. Glanzmann thrombasthenia and its perioperative management in head trauma: $\mathrm{A}$ rare and challenging situation. J Neuroanaesthesiol Crit Care 2016;3:52-5.

Source of Support: Nil, Conflict of Interest: None declared. 